\title{
QUANTUM ASPECTS OF ACCRETION ONTO BLACK HOLES IN THE EARLY UNIVERSE*
}

\author{
S. W. HAWKING and G. W. GIBBONS \\ Institute of Astronomy, Cambridge, England
}

\begin{abstract}
It is argued that for black holes of mass less than roughly $10^{17} \mathrm{~g}$ formed in the early Universe, the accretion process must be calculated using quantum mechanics. It is also argued that primordial black holes must be more massive than $10^{17} \mathrm{~g}$ if they are to possess charge.
\end{abstract}

* To be published. 